\title{
AGING AND THE DETECTION OF COLLISION EVENTS IN FOG
}

\author{
Zheng Bian ${ }^{1}$, Rui $\mathrm{Ni}^{2}$, Amy Guindon ${ }^{1}$ and George J. Andersen ${ }^{1}$ \\ ${ }^{1}$ University of California, Riverside \\ Department of Psychology \\ ${ }^{2}$ Wichita State University \\ Email: andersen@ucr.edu
}

\begin{abstract}
Summary: The current study investigated age-related differences in the detection of collision events in fog. Observers were presented with displays simulating an object moving towards a driver at a constant speed and linear trajectory. The observers' task was to detect whether the object would collide with them. Fog and display duration of the object were manipulated. We found that performance decreased when fog was simulated for older but not for younger observers. An age-related decrement was also found with shorter display durations. These results suggest that under poor weather conditions with reduced visibility, such as fog, older drivers may have increased accident risk due to decreased ability to detect impending collision events.
\end{abstract}

\section{INTRODUCTION}

An important perceptual task in driving is to detect collision events. Accurate detection of an impending collision is the basis of making appropriate responses, such as steering to avoid a collision. There are various situations under which an observer may collide with an object, depending on their motion (Andersen \& Sauer, 2004). For instance, Andersen et. al. (1999) examined the detection of collision events when observers were decelerating to a stationary object. They found that observers' sensitivity was affected by the perceived speed, the size of the object, and the edge rate information. Older observers were less sensitive to impending collision events than younger observers, and the sensitivity was affected by the simulated speed of observers and the edge rate information (Andersen et. al., 2000). DeLucia et. al. (2003) studied the age-related differences in detecting collision events when an object was moving towards the observer at a constant speed. They found an age-related decrement in sensitivity for older females but not older males. Finally, Andersen and Enriquez (2006) found that older observers, as compared to younger observers, were less sensitive to collision events with increased speed, shorter display duration and longer time-to-contact. In the current study, we examined agerelated differences in detecting collision events when an object was approaching the observer at a constant speed on a linear trajectory with fog.

Fog is a weather condition that reduces contrast in a scene. Fog usually forms at night and lasts until morning hours when traffic is heavy, and thus may lead to higher injury and death rates in accidents (Al-Ghamdi, 2007). For instance, in 1998 two major fog-related crashes occurred within 3 weeks on I-64 in the Afton Mountain area in Virginia. One crash involved 65 cars while the other crash involved 21 cars, resulting in more than 40 injuries (Lynn et. al., 2002). Driving simulation studies have found evidence of decreased driving performance under simulated fog conditions. Previous studies have shown that fog decreased the judged distance between an observer and a leading vehicle (Kang et al., 2008). Performance of detecting traffic light changes 
and car following tasks also decreased with fog density, especially for older observers (Ni \& Andersen, 2007). In the current study, we examined the age-related differences in collision detection with fog. An important characteristic of fog is that it reduces contrast of the driving scene as a function of distance. Previous research has found decreased contrast sensitivity with age (Owesley, Sekuler \& Seimsen, 1983; Elliot, 1987). These finding suggest that older observers may have more difficulty than younger observers in recovering visual information needed to correctly detect impending collision events under foggy conditions.

\section{EXPERIMENT}

\section{Methods}

Observers. 11 younger observers (mean age $=23$ ) and 11 older observers (mean age $=75$ ) participated in a collision detection study. All observers had a minimum of 2 years of driving experience, had normal or corrected to normal vision, and were naïve to the purpose of the experiment.

Apparatus. The displays were presented on a 23-inch flat screen LCD monitor with a pixel resolution of 1600 by 1200 at a refresh rate of $60 \mathrm{~Hz}$, controlled by a Windows XP Professional Operating System. The visual angle was approximately $66^{\circ}$ by $51^{\circ}$. A $19-\mathrm{cm}$ diameter collimating glass lens, which magnified the images by approximately $19 \%$, was located between the observer and the monitor. The purpose of the collimating lens was to remove differences in accommodative focus between younger and older observers. Observers viewed the displays binocularly at a distance of approximately $45 \mathrm{~cm}$ from the screen.

Stimuli. The displays simulated 3-D scenes consisted of a roadway with a solid double-yellow line. The width of the roadway was $3.6 \mathrm{~m}$. Textured green grass was projected on both sides of the roadway (see Figure 1). Within the scene there was a bright purple sphere with a simulated diameter of $3.6 \mathrm{~m}$ translating towards the observer at a speed of $90 \mathrm{~km} / \mathrm{h}(56 \mathrm{~m} / \mathrm{h})$ from a distance of $223 \mathrm{~m}$. The initial position of the sphere was chosen randomly from an arc with a radius of $223 \mathrm{~m}$ centering the observer within the field of view. The projected size of the sphere varied as a function of distance, with the smallest projected size as $0.9^{\circ}$. The average luminance was 12.06 $\mathrm{cd} / \mathrm{m}^{2}$ for the no fog condition, and $19.13 \mathrm{~cd} / \mathrm{m}^{2}$ for the fog condition. The contrast of the object at the beginning of the motion was 0.04 for the no fog condition and 0.01 for the fog condition. The contrast of the object at the end of motion for no fog condition was 0.04, 0.06 and 0.13 , respectively for the display duration of 3, 5, and 7 seconds. The contrast of the object at the end of motion for fog condition was 0.01 for all display durations. All observers reported during debriefing that they were able to see the sphere in all conditions.

Design. The 3-way mixed design included age as the between-subject variable and 2 withinsubject variables: (1) fog density (no fog or dense fog); (2) display duration of the sphere $(3,5$, or 7 seconds). The within-subject variables produced 6 combinations, each of which was repeated 40 times in the experiment (20 times with a collision event simulated and 20 times with a non-collision event simulated), producing a total of 240 trials. The 240 trials were divided evenly into 2 blocks, which were completed within one single-hour session. These experimental 
blocks were preceded by a 16-trial practice block. The order of the trials for each observer in each block was randomized individually.

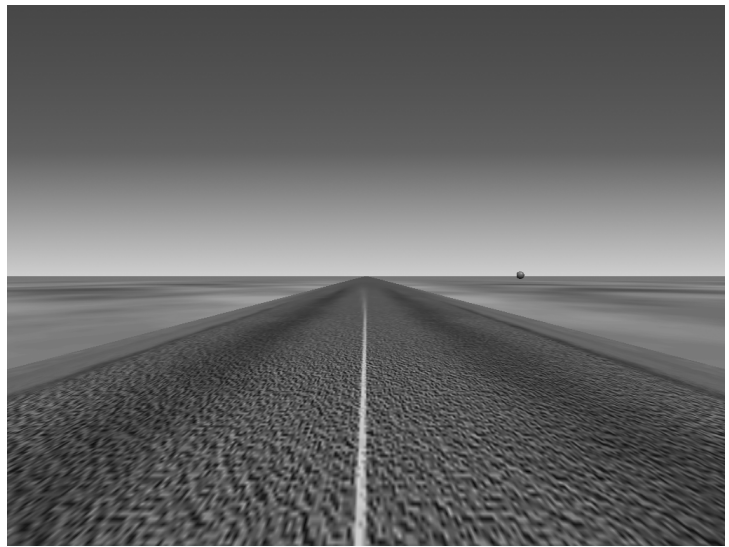

a. No Fog

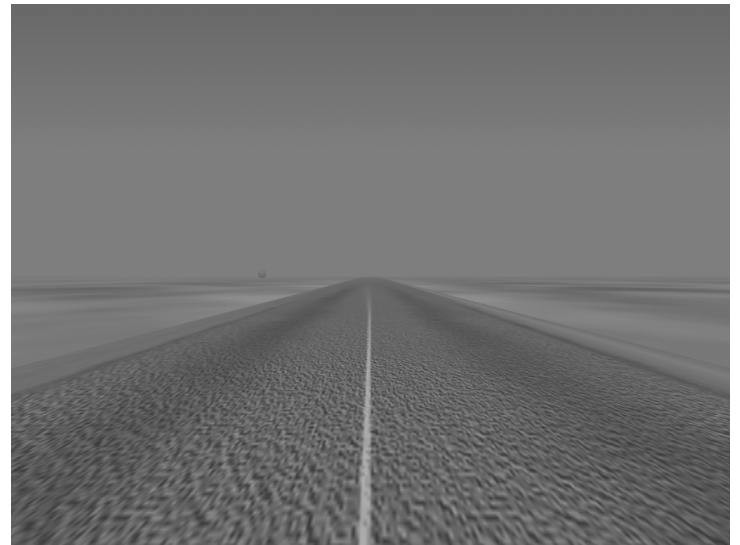

b. Dense Fog

Figure 1. Example of stimuli with different fog conditions

Procedure. The experiment was run in a darkened room. The observer viewed the monitor binocularly through a collimating lens with their head in a chin rest. On each trial, a scene consisting of a roadway and a sphere was presented. The sphere traveled from distance to the observer at a constant speed and disappeared after sometime before reaching the observer plane. The task of the observer was to determine whether the sphere would collide with the observer (a collision event) or pass the observer (a non-collision event). Observers responded by pressing either the ' 4 ' button (collision) or the ' 6 ' button (non-collision) on a standard keyboard. Examples of the complete collision and non-collision events were shown to the observers during instructions. A practice block with partial motion paths of collision and non-collision events (i.e., observers did not see the object fill the screen or pass outside the field of view) was conducted followed by experimental blocks. No feedback was given during the experiment.

\section{RESULTS}

We calculated the proportion of hit and false alarms for each observer and derived a sensitivity $\left(d^{\prime}\right)$ and bias $(\beta)$ score (Green \& Swets, 1966) for each condition. In conditions where observers performed perfectly (100\% hit rate or $0 \%$ false alarm rate), $d$ ' and $\beta$ were calculated assuming that one error was made. A 3-way mixed ANOVA with repeated measures was conducted for both d' and $\beta$. Greenhouse-Geisser adjusted $p$-values were reported when sphericity was violated (Greenhouse \& Geisser, 1959), although the actual (rather than the adjusted) degrees of freedom were always reported.

Sensitivity. Overall we found a main effect of age $(\mathrm{F}(1,20)=6.07, \mathrm{MSE}=1.65, \mathrm{p}<.05)$, indicating that younger observers (mean $d^{\prime}=3.01$ ) had greater sensitivity than older observers (mean $\left.\mathrm{d}^{\prime}=2.45\right)$. The main effect of fog was found to be significant $(\mathrm{F}(1,20)=11.44, \mathrm{MSE}=$ $0.09, \mathrm{p}<.01)$, as well as the interaction of age and $\mathrm{fog}(\mathrm{F}(1,20)=9.72, \mathrm{MSE}=0.09, \mathrm{p}<.01)$. 
As can be seen in Fig 2, the presence of fog resulted in decreased sensitivity for older observers but not for younger observers.

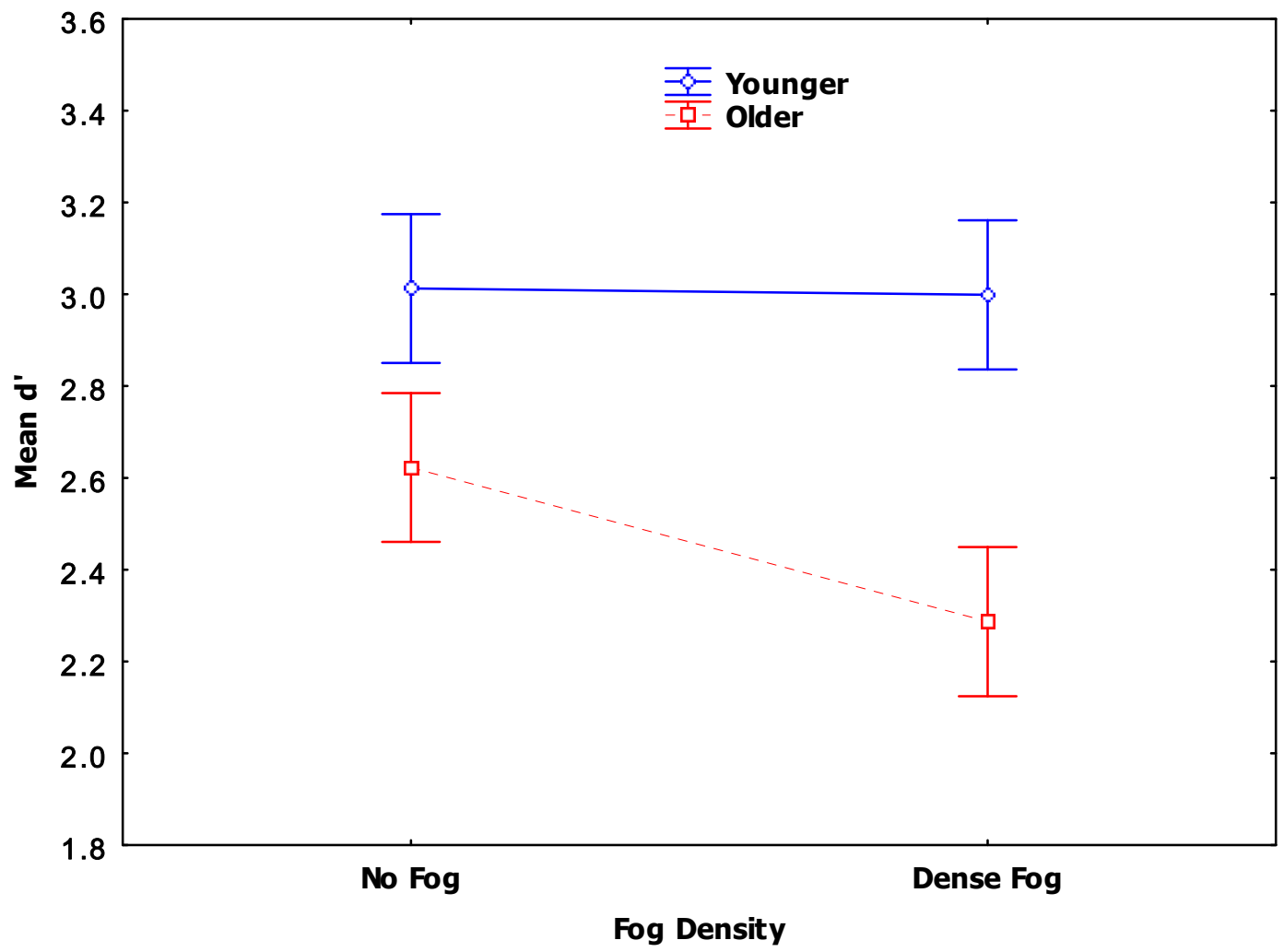

Figure 2. The interaction between age and fog density on sensitivity (d')

The main effect of display duration of the object motion was significant $(\mathrm{F}(2,40)=34.67$, MSE $=0.48, \mathrm{p}<.01)$, which was mediated by a significant interaction with age $(\mathrm{F}(2,40)=6.23$, MSE $=0.48, \mathrm{p}<.01)$. When the display duration was 7 seconds, older observers performed as well as younger observers. As the display duration decreased, the sensitivity for both age groups decreased. However, older observers' sensitivity decreased much faster than that of younger observers (Fig 3). The 3-way interaction between age, fog, and display duration was not significant $(\mathrm{F}(2,40)=0.94, \mathrm{MSE}=0.11, \mathrm{p}=.40)$, nor were any other effects significant $(\mathrm{p}>.05)$.

A linear regression between contrast sensitivity and the sensitivity of collision detection in fog was conducted for both age groups to examine whether the sensitivity of detecting collision events was predicted by contrast sensitivity. The results indicated that contrast sensitivity was not a significant predictor for sensitivity of collision detection for either younger observers $\left(\mathrm{r}^{2}=\right.$ $0.09, \mathrm{p}=0.37)$ or older observers $\left(\mathrm{r}^{2}=0.06, \mathrm{p}=0.46\right)$. There are two explanations which might account for this result. First, the contrast sensitivity for each observer was measured using Pelli Robson test (Pelli, Robson, \& Wilkins, 1988), which may not have sufficient precision to assess individual differences. For example, 10 out of 11 younger observers in this study had the same contrast sensitivity whereas their d' ranged from 2.38 to 3.24. An important issue for future research would be to precisely derive contrast sensitivity thresholds. Second, the Pelli Robson test only measured contrast sensitivity in static scenes whereas our stimuli were in motion. Observers might have different contrast sensitivity when viewing motion stimuli as compared to 
when viewing static stimuli. A similar result has been found for studies examining sensitivity to binocular disparity (Braunstein, Andersen, Rouse \& Tittle, 1986). Specifically this research has found that observers who were stereo-blind using static stimuli had sensitivity to disparity when the stimuli were moving. If similar conditions exist for low contrast stimuli then the assessment of contrast sensitivity with static stimuli would not be predictive of sensitivity to low contrast moving stimuli.

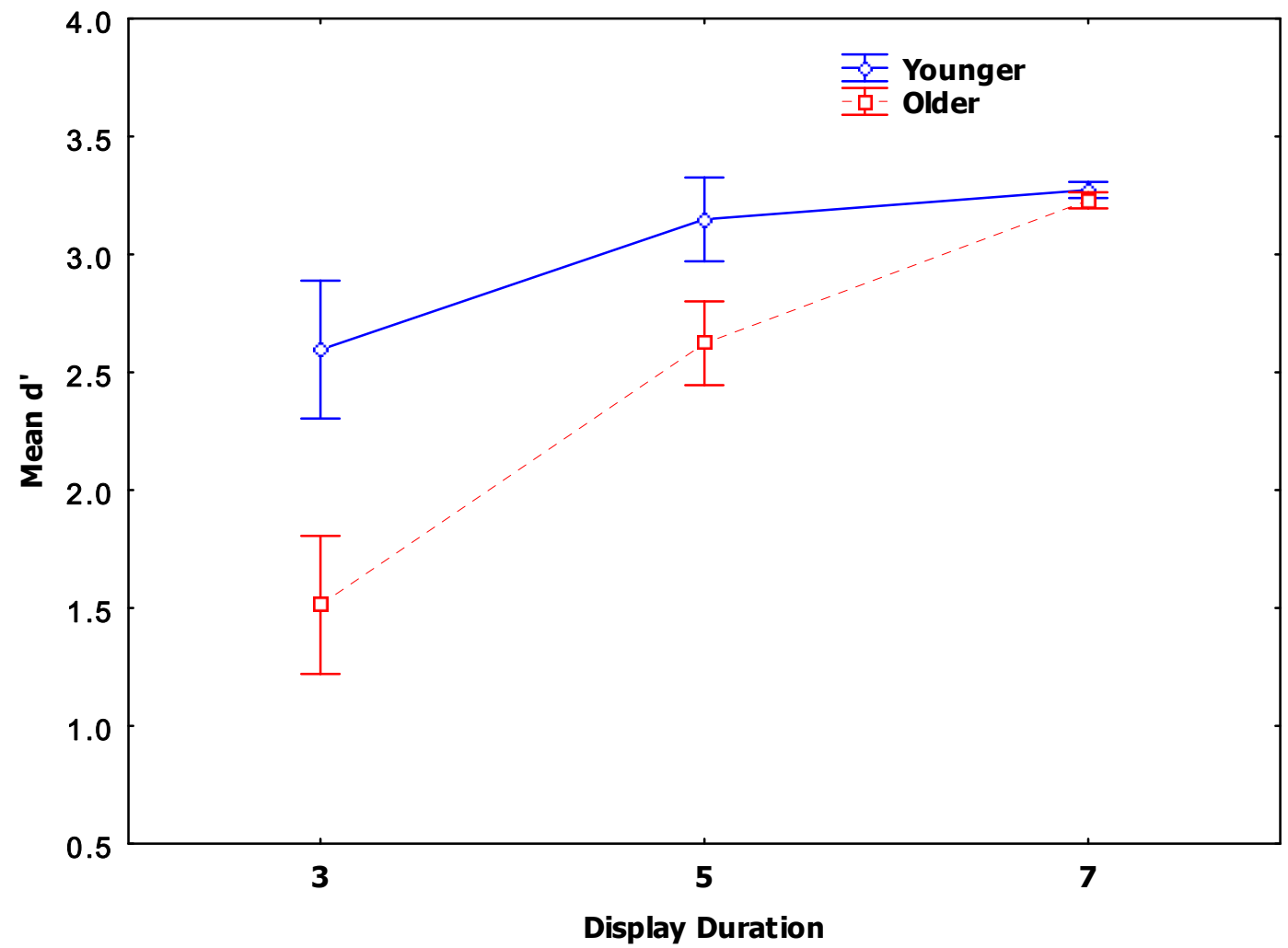

Figure 3. The interaction between age and display duration on sensitivity (d')

An important issue is whether the age-related decrements in collision detection were due to an inability of older observers to perform the task. To examine this issue we conducted an additional analysis to determine if the d' values for each observer in each condition were significantly greater than zero (chance) using a test proposed by Marascuilo (1970). Younger observers' sensitivity was always significantly greater than zero $(p<.05)$ in all 6 combinations of fog and display duration. For the no fog displays the number of older observers with greater than zero sensitivity was 8,10 , and 11 (out of 11 ) for the $3 \mathrm{~s}, 5 \mathrm{~s}$, and $7 \mathrm{~s}$ display duration, respectively. For the fog displays the number of older observers with greater than zero sensitivity was 6,11 , and 11 (out of 11) for the $3 \mathrm{~s}, 5 \mathrm{~s}$, and $7 \mathrm{~s}$ display duration, respectively. These results suggest that the age-related decrements in collision detection with fog at the 7 and 5 seconds of display duration were due to decreased sensitivity to detect collision events rather than due to inability to perform the collision detection task. For the 3-second display duration, about half of the older observers were not able to significantly detect collisions with better than chance performance when fog was simulated. 
Bias. The main effect of age group was not significant $(\mathrm{F}(1,20)=0.31, \mathrm{MSE}=0.94, \mathrm{p}=.58)$, suggesting that both age groups were equally likely to make a collision judgment. A significant main effect of fog $(\mathrm{F}(1,20)=5.05, \mathrm{MSE}=0.10, \mathrm{p}<.05)$ and display duration of the stimuli $(\mathrm{F}(2$, $40)=9.82, \mathrm{MSE}=0.40, \mathrm{p}<.05)$ were found. No other significant effect was found. According to these results, when fog was simulated or when the display duration decreased, observers were more likely to respond that a collision would occur.

\section{DISCUSSION}

In this study, we examined age-related differences in the detection of collision events under fog conditions. We predicted that when fog was simulated, both younger and older observers would show decreased sensitivity but older observers would show a greater decrement because of decreased contrast sensitivity. The results for younger observers indicate that detection sensitivity did not vary as a function of the presence/absence of fog. Older observers, however, showed a decreased sensitivity to detect a collision event when fog was present. An age-related decrement was also found with shorter display durations, a finding consistent with previous research (Andersen \& Enriquez, 2006). This result is probably due to the increased difficulty in discriminating expansion information when the magnitude of expansion is small. Considered together, these results suggest that under poor weather conditions, such as fog, older drivers may be subject to an increased crash risk due to a decreased ability to detect impending collision events.

The current study adds to the growing body of literature showing that the decreased ability in processing perceptual information accompanying aging may lead to increased risk of older drivers (e.g., Andersen et. al., 2000; Andersen \& Enriquez, 2006; De Lucia, et. al. 2003). An important issue for future research is whether this decrement can be minimized or even reversed through training. Ni et al. (2007) found that following training with subthreshold stimuli older observers were able to improve performance in a texture discrimination task. Indeed, the training, which occurred over a 2 day period, resulted in performance equal to the performance of college age observers. Richards et. al. (2006) found that older observers could improve their performance in divided attention through repeated training with the UFOV (useful field of view) task, and that this improvement was retained for up to 3 months. An interesting topic for future research would be to investigate whether older observers can improve collision detection performance through the use of training techniques.

\section{ACKNOWLEDGMENT}

This research was supported by NIH grant AG13419-06 and NIH EY18334-01.

\section{REFERENCES}

Al-Ghamdi, A. S. (2007). Experimental evaluation of fog warning system. Accident Analysis and Prevention. 39, 1065-1072.

Andersen, G. J., Cisneros, J., Atchley, P., \& Saidpour, A. (1999). Speed, size, and edge-rate information for the detection of collision events. Journal of Experimental Psychology: Human Perception and Performance. 25, 256-269. 
Andersen, G. J., Cisneros, J., Saidpour, A., \& Atchley, P. (2000). Age-related differences in collision detection during deceleration. Psychology and Aging, 15, 241-252.

Andersen, G. J., \& Enriquez, A. (2006a). Aging and the detection of observer and moving object collisions. Psychology and Aging, 21, 74-85.

Braunstein, M. L., Andersen, G. J., Rouse, M. W., \& Tittle, J. S. (1986). Recovering viewercentered depth from disparity, occlusion, and velocity gradients. Perception \& Psychophysics, 40, 216-224.

DeLucia, P. R., Bleckley, M. K., Meyer, L. E., \& Bush, J. M. (2003). Judgments about collisions in younger and older drivers. Transportation Research part F: Traffic Psychology \& Behavior, 6, 63-80.

DeLucia, P. R., \& Warren, R. (1994). Pictorial and motion-based depth information during active control of self-motion: Size-arrival effects on collision avoidance. Journal of Experimental Psychology: Human Perception \& Performance, 20, 783-798.

Greenhouse, S., \& Geisser, S. (1959). On methods in the analysis of profile data. Psychometrika, 24, 95-112.

Kang, J. J., Ni , R., \& Andersen, G. J. (2008). The effects of reduced visibility from fog on car following performance. Transportation Research Record: Journal of the Transportation Research Board, 2069, 9-15.

Lynn, C., Schreiner, C., Campbell, R. (2002) Reducing fog-related crashes on the Afton and Fancy Gap Mountain sections of I-64 and I-77 in Virginia. Report FHWA/VTRC 03-CR2. Virginia Transportation Research Center, Charlottesville.

Marascuilo, L. A. (1970). Extensions of the significance test for one parameter signal detection hypotheses. Psychometrika, 35, 237-243.

Owsley, C., Sekuler, R., \& Siemsen, D. (1983). Contrast sensitivity throughout adulthood. Vision Research, 23, 689-699.

Ni, R., Watanabe, T., \& Andersen, G. J. (2007). The effect of age on perceptual learning of subthreshold stimuli [Abstract]. Journal of Vision, 7(9):47, 47a, http://journalofvision.org/7/9/47/, doi:10.1167/7.9.47.

Ni, R., Kang, J., \& Andersen, G. J. (2007). Age-related driving performance: effect of fog under dual-task conditions. Proceedings of the Fourth International Driving Symposium on Human Factors in Driver Assessment, Training and Vehicle Design, 365-370.

Pelli, D.G., Robson, J.G., \& Wilkins, A, J. (1988). The design of a new letter chart for measuring contrast sensitivity. Clinical Vision Science, 2, 187-199.

Richards, E., Bennett, P. J., Sekuler, A. B. (2006) Age related differences in learning with the useful field of view. Vision Research. 46, 4217-4231 